\title{
Les journaux de séjour de Stendhal et Faure à Vienne en 1809 : images de la ville et images de soi
}

Muriel Bassou

\section{(2) OpenEdition}

Journals

Édition électronique

URL : https://journals.openedition.org/recherchestravaux/476

DOI : 10.4000/recherchestravaux.476

ISSN : 1969-6434

Éditeur

UGA Éditions/Université Grenoble Alpes

\section{Édition imprimée}

Date de publication : 15 décembre 2011

Pagination : 73-95

ISBN : 978-2-84310-215-8

ISSN : 0151-1874

\section{Référence électronique}

Muriel Bassou, « Les journaux de séjour de Stendhal et Faure à Vienne en 1809 : images de la ville et images de soi », Recherches \& Travaux [En ligne], 79 | 2011, mis en ligne le 15 juin 2013, consulté le 07 décembre 2022. URL : http://journals.openedition.org/recherchestravaux/476 ; DOI : https://doi.org/ 10.4000/recherchestravaux.476 


\section{Les journaux de séjour de Stendhal et Faure à Vienne en 1809 : images de la ville et images de soi}

Lobjet journal de voyage est difficile à cerner. Gérald Rannaud le soulignait déjà dans son article "Le journal de voyage : forme littéraire ou fait culturel?» en 1986. Aux deux extrêmes, il distinguait deux formes assez pures, bornes entre lesquelles tous les cas sont possibles : le journal de bord «tenu au jour le jour par un scripteur dont le personnage s'abolit ou se confond dans la matérialité du voyage ${ }^{\mathrm{I}}$ " et le journal intime "où le scripteur prévaut sur le voyage» et "où le voyage n'[aurait] d'autre valeur qu'incidente ${ }^{2} »$. Cet immense entre-deux semble laisser peu de place à l'invention de formes nouvelles. Et pourtant, on peut imager un journal de séjour, miroir inversé de la mobilité du journal de voyage. La formule n'est pas de notre cru : Stendhal intitule un de ses cahiers "I8I4. Journal de mon triste séjour à Grenoble»" et entame avant de partir pour Strasbourg un journal intitulé «Mon séjour à Paris en $1809^{4} »$. Mais le journal de séjour est-il une forme de journal suffisamment défini pour qualifier n'importe quel séjour?

Arrivé à Vienne le 13 mai I809, Stendhal reste six mois au service de Martial Daru, nommé intendant de la province. Pas de doute, il envisage bien sa vie

I. G. Rannaud, «Le journal de voyage : forme littéraire ou fait culturel?», dans Le Journal de voyage et Stendhal (actes du colloque de Grenoble de 1983), textes recueillis par V. Del Litto et E. Kanceff, Genève, Slatkine, 1986, p. I37.

2. Ibid.

3. Journal, éd. V. Del Litto, dans CEuvres intimes, t. I, Gallimard, coll. «Bibliothèque de la Pléiade», I98I, p. 90I. Nous abrégerons désormais cette édition en $J$.

4. J, p. 5I4. 
à Vienne comme un «séjour», et une fois sur place, il l'envisage même avec sérénité : «me voilà Viennois pour un an ou deux ». Le terme se rencontre à la fois dans la première et la dernière des lettres qu'il envoie à cette période-là. Il écrit à Faure le I8 mai : "J'ai éprouvé, les premiers jours de mon séjour à Vienne, ce contentement intérieur et bien-être parfait que Genève seule m'avait rappelé depuis l'Italie ${ }^{6} . »$ Et il raconte à Pauline comment, alors qu'il est revenu à Vienne pour l'entrée de François II, il a lancé gaillardement à l'adresse de Madame Salmi "la plus belle femme de la ville, dit-on» : "Il est heureux pour moi de voir, le dernier jour de mon séjour à Vienne, la femme la plus belle et l'événement le plus remarquable 7 .» Mais pendant les six mois où il y séjourne, Stendhal ne rédige un journal en bonne et due forme que pour une période bien restreinte, celle du 2I octobre au 20 novembre. C'est que ce journal est moins celui de son propre séjour que celui d'une autre personne $^{8}$, celle qui va trouver en ce lieu un de ses nouveaux visages: Madame Palfy', autrement dit Alexandrine Daru. Aussi notre hypothèse de lecture est-elle de considérer l'ensemble des lettres viennoises de Stendhal comme son second journal de séjour dont tout l'intérêt réside dans sa différence de statut générique. Celui-ci, composé de onze lettres (dix à Pauline et une seule à Faure $^{\mathrm{r}}$ ), couvre, cette fois, l'ensemble du séjour de Stendhal (du I8 mai au 29 novembre). Enfin, notre réflexion va englober un dernier journal de séjour qui apporte un regard croisé sur le journal stendhalien : il s'agit de celui de Faure qui vient passer un mois à Vienne (du 2 août au 5 septembre). Il évoque lui aussi «[s]on séjour ${ }^{\mathrm{II}} »$ dans la ville. Signalons que ce journal, qu'on

5. À Félix Faure, I8 mai I809, Correspondance générale, t. I., éd. V. Del Litto, Honoré Champion, 1997, p. 833. Nous abrégerons désormais cette édition en $C G$.

6. Ibid.

7. À Pauline, Vienne, 29 novembre $1809, C G$.

8. Dans un texte postérieur rédigé en collaboration par Stendhal et Crozet, «La Consultation pour Banti», les deux amis revenant sur l'épisode viennois évoqueront le séjour de la duchesse dans la capitale autrichienne, tout en dilatant la chronologie : il ne sera plus question d'un, mais de «trois mois»; façon comme une autre de donner plus d'importance à cette histoire avortée (J, p. I069-I070).

9. «Le nom Palfy est emprunté à celui d'une des familles les plus connues de Vienne. [...] En adoptant ce pseudonyme, Stendhal a voulu dater en quelque sorte sa rencontre à Vienne avec Mme Daru.» (Note de V. Del Litto, Euvres intimes, t. I, coll. «Bibliothèque de la Pléiade», Gallimard, I989, p. I345). Nous abrégerons désormais cette édition en $O I$.

Io. Pendant la "Campagne de Vienne», Stendhal avait écrit sept lettres à Faure et trois à Pauline (entre le 5 avril et le II mai à Sankt-Pölten), il lui renouvelle ce jour-là toute son amitié dans une formule pleine de nostalgie : «Je te regrette bien depuis quelques jours; il me semble qu'il y a un an que j'ai quitté Paris. (À Félix Faure, Sankt-Pölten, le II mai ı8o9, CG, p. 833) Dès lors, on peut se demander d'où vient tout à coup cette absence de lettres. C'est sans doute parce que son ami est à son tour sur les routes; de fait, le voyage complet de Faure aller et retour dure deux mois et demi, du 20 juillet au 4 octobre.

II. 28 août I809, J, p. I055. 
trouve dans les annexes de l'édition des CEuvres intimes de Stendhal, n'est qu'un extrait d'un plus ample journal de voyage ${ }^{\text {I2 }}$ (Faure part le 20 juillet de Paris et rentre à Saint-Ismier le 4 octobre). Il est intéressant de réfléchir au statut particulier de ce passage du journal, isolé, dans l'édition de Victor Del Litto, au sein de l'ensemble auquel il appartient ${ }^{13}$. Un tel corpus permet de croiser à la fois deux regards sur la ville et deux approches génériques. Or, au cœur de ces questions, se pose à chaque fois une problématique unique : celle de l'ouverture, de la circulation, du dialogisme. De fait, le journal de Faure sera lu et commenté par Stendhal; celui de Stendhal est a priori destiné à une relecture personnelle visant à analyser sa conduite mais semble faire l'objet d'une étude conjointe avec un autre ami, Crozet, dans un texte ultérieur : «La Consultation pour Banti»; quant à la correspondance, inutile de préciser sa visée dialogique si ce n'est pour souligner le redoublement qui peut avoir lieu en son sein (rôle d'archive, rôle de relais postal).

À travers l'étude de ces trois exemples, aussi différents soient-ils, la question qui se pose est celle de la représentation d'un concept qui est à définir - celui de journal de séjour. Pour cela, il faudra nous interroger sur le rapport au temps et au lieu de cette écriture diariste. Comme socle de notre réflexion nous commencerons par comparer le journal de séjour au journal de voyage en prenant comme point de comparaison à nos trois journaux la «Campagne de Vienne». Mais cette approche globalisante ne tient pas compte des spécificités des journaux de séjour de notre corpus qui se répondent deux à deux. Comment fonctionnent donc le dédoublement du journal de séjour stendhalien et l'imperméabilité des écritures diaristes et épistolaires? De même, comment les journaux de séjour de Stendhal et de Faure offrent-ils deux miroirs croisés de la Ville et du Moi?

I2. On peut en trouver des extraits dans le livre de J. Félix-Faure, Un compagnon de Stendhal, Félix Faure, pair de France, chap. Ix "Le voyage en Autriche», Aran (Suisse), Éditions du Grand Chêne, "Collection stendhalienne», I978.

I3. Victor Del Litto explique qu'il ne s'agit pas de la rédaction originale de ce voyage, mais de la copie que Faure a exécutée en 1852 et dont on peut se demander, à juste titre, si, plus qu'une copie, elle n'est pas la version réécrite du journal originel. Au début de son journal, daté du 28 août I809, on trouve une analepse qualifiée : «Du I4 au 28 août, mon genre de vie a été à peu près le même : me promenant, observant, lisant, et le seul événement remarquable a été une chute de cheval [...] dont l'effet a été une foulure au pouce droit qui a été plus de deux mois à se guérir. [...] Ceci a un peu nui à mon journal. [...] Aujourd'hui, mon pouce va mieux $[\ldots]$ » (J, p. I055). Comment le diariste peut-il prédire le jour même le temps exact de guérison de sa foulure? 
Recherches \& Travaux $-\mathrm{n}^{\circ} 79$

\section{Journal de voyage et journal de séjour : l'invention d'une forme?}

Il semble qu'il y ait, pour Stendhal, une interdépendance entre la notion de séjour et celle de voyage. Dans son journal de I8or, figure ce passage :

Je suis né le 23 janvier 1783 , à Grenoble, rue [des] Vieux-Jésuites. Je suis parti pour Paris, le 8 brumaire an VIII. J'y suis arrivé le I9 du même mois. J'en suis parti, après cinq mois et vingt-huit jours de séjour, le 17 floréal ${ }^{14}$.

De cet extrait, nous pouvons déduire : I) qu'un séjour a lieu entre deux voyages: "j'y suis arrivé, j'en suis parti»; 2) qu'un séjour a une durée déterminée : «après cinq mois et vingt-huit jours»; 3) qu'un séjour se déroule dans un lieu précis, en l'occurrence Paris, pour le jeune Grenoblois ${ }^{15}$. Or, le Trésor de la langue française informatisé donne les définitions suivantes à l'article "séjour»: I) "le fait de demeurer un certain temps dans un lieu»; 2) «la durée pendant laquelle quelqu'un demeure dans un lieu»;3) «le lieu où l'on demeure pendant un certain temps ${ }^{16} »$. Un hiatus est donc visible : dans le dictionnaire n'apparaît pas l'avant et l'après du séjour, le mouvement qui conduit à la pause, l'action qui amène le fait. Or, c'est cette interdépendance qui semble essentielle chez Stendhal. C'est en ce sens qu'on peut comprendre le titre du fameux "Journal de mon troisième voyage à Paris» qu'on ne retrouve pas moins d'une douzaine de fois sur ses cahiers couvrant la période d'avril I804 à avril I80 $5^{17}$. Dans l'un de ceux-ci - "Troisième voyage à Paris. Journal du I8 germinal au 30 floréal exclusiv[ement]» - on lit, à la date du I9 germinal an XII [9 avril I804] : "Je me trouve plus raisonnable qu'à mon dernier séjour et, par conséquent, je serai plus heureux; je dois cela à l'expérience acquise à Gr[enoble], où j'ai vu l'homme dans l'homme et non plus dans les livres ${ }^{18}$.»

C'est par rapport au point d'ancrage que représente la maison du pater familias que Stendhal peut évoquer Paris comme une ville où il séjourne entre deux voyages. D'ailleurs, la vie de Stendhal dans la capitale pourrait être envisagée comme un séjour qui réactualiserait des sens du mot aujourd'hui disparus : estre en sejor voulait dire "être oisif» et estre a sejor "être de repos dans un lieu agréable». Pour Stendhal, Grenoble ne sera pas toujours le «terme

I4. J, p. 32, 29 [frimaire an $\mathrm{X}: 20$ décembre I8oI].

I5. Un séjour peut avoir une durée très variable. Durant la "Campagne de Vienne", Stendhal s'arrête quelques jours dans une ville et parle immédiatement de "séjour»; "Rien de nouveau pendant les deux derniers jours de mon séjour à Ingolstadt (les 20 et 2I). (Landshut, le 23 avril [1809], J, p 525) Voir aussi le "Journal contenant ce que j'ai fait pendant les douze ou quinze derniers jours du séjour de mon oncle à Paris.» (J, p. I69)

I6. TLFi (http://www.atilf.atif.fr), entrée «Séjour».

I7. Voir $J$, p. 78, 95, 97, II2, I37, I65, I94, 217, 228, 284, 30I, 314.

I8. I9 germinal [an XII : 9 avril I804], J, p. 58. 
d'arrivée», ce «lieu d'enracinement» où "on laisse ses possessions, ses attaches, [où] on conserve des droits", ce lieu où «il est bien entendu depuis le début qu'on va revenir ${ }^{19}{ }^{\text {", }}$ pour reprendre les analyses que développe Butor dans son "itérologie ${ }^{20}$ ». Loin s'en faut! Grenoble sera aussi considérée comme une ville où l'on fait un "triste séjour» quand Stendhal y est envoyé pour une mission militaire. Il écrit à ce sujet : «Comment décrire sans renouveler mon apathie et mon ennui les cinquante-deux jours que j'ai passés dans ce quartier général de la petitesse $e^{21}$ ?" Mais si le séjour ne semble pouvoir se définir que par le concept de voyage, en est-il de même pour les journaux de voyage et de séjour?

\section{"L'absence de frontière entre journal intime et journal de voyage ${ }^{22}$ "}

Selon Sabine Ceysson, il y aurait chez Stendhal, "une sorte de coïncidence poétique entre journal intime et journal de voyage. Son Journal se présente en effet comme celui de ses nombreux déplacements ${ }^{23} »$. De fait, si l'on observe les titres de ses différents journaux ${ }^{24}$, on s'aperçoit de la large part donnée aux journaux de voyage ${ }^{25}$. Citons entre autres, "Travel in Grenoble in the spring of $I 805^{26 "}$, "Journal du voyage à la mer. Partis le 29 avril, de retour le $3 \mathrm{mai}^{27}$ ", "A journal of a tour to Venezia and Padova, (June I8I5)"28", "Journal du voyage dans la Brianza (août $1818^{29}$ )", "Journal d'un voyage à Londres en $1817^{30}$ ". On peut aussi mettre dans cette catégorie son "A tour through some parts of Italy in the year $I 8 I I^{3 \mathrm{I}} »$. D'autres journaux sont également écrits sur les

19. M. Butor, "Le voyage et l'écriture», Romantisme. Revue de la Société des Études romantiques, $\mathrm{n}^{\circ}$ 4, Flammarion, 1972, p. IO.

20. Ibid., p. 7.

2I. J, p. 902.

22. V. Del Litto, "Journal de voyage et journal intime chez Stendhal », dans Le Journal de voyage et Stendhal, ouvr. cité, p. 9.

23. S. Ceysson, "Le "journal intime" de Stendhal», dans Stendhal hors du roman, textes réunis par D. Sangsue, Dijon, université de Bourgogne, Actes, nº 9, 200I, p. 5I.

24. Notre relevé ne se prétend pas exhaustif.

25. Relevé qui ne tient pas compte des innombrables petits récits de voyage à l'intérieur des journaux eux-mêmes : «Voyage à Twülpstedt» (p. 477) (avec Strombeck et alii); "Voyage au Brocken» (Io juillet I807, p. 483-485).

26. J, p. 33I.

27. J, p. 675 .

28. J, p. 934.

29. J, t. II, p. I2.

30. $J$, p. 1098.

31. J, p. 655. Voir les autres mentions du titre "A tour through Italy. I8II» (J, p. 715) et «Fin d'octobre I8II. The last part of a tour throught Italie présenté en toute humilité à M. H[enri de Beyle] âgé de 39 ans, qui vivra peut-être en I82I, par son très humble serviteur plus gai que lui, le H[enri B[eyle] de I8II. Milan, le 29 octobre I8II.» ( $J$, p. 8I3) 
routes, tels les journaux de Campagne : "Campagne de Vienne en I80932", "Campagne de I809 de Strasbourg à Vienne ${ }^{33}$ » (journal avorté), "Journal de ma Campagne de Russie en $1812^{34}$ », "Fragment du journal de la Campagne de I8I3 (en original). Intendance à Sagan, 7 juin $1813^{35}$ ». Restent ensuite des journaux au statut générique plus flou mais dont le titre traduit l'orientation que Stendhal assigne principalement à l'écriture diariste, à savoir l'examen de soi ; plutôt que de parler de journaux «intimes», préférons l'idée de journaux d'analyse du moi. Ainsi des journaux rédigés à Marseille : "Journal de sa vie. Du 9 thermidor an XIII [28 juillet I805] jusqu'au Is avril I806] ${ }^{36}{ }_{n}$; d'un journal rédigé à Paris en I812 «Something for the life of Myself7» ou des journaux d'analyse de ses relations avec Madame Daru : "Djorn'l, or anatomy, of the thougts, feeling and events of Harry, from the gth May I8ro till the I2 aout I8IO. ( 3 mois et 3 jours) ${ }^{38}$." D'autres journaux sont souvent simplement datés : "Journal du I7 juin I807 au [un blanc]. I807-I80839" qui sera celui de son séjour à Brunswick; ou encore "Journal, I8I4, $6^{\mathrm{e}}$ cahier $^{40}$ " (il est alors en Italietri) etc. Mais cette classification à partir des titres ne permet pas de rendre compte du brouillage des formes d'écriture diariste chez Stendhal ${ }^{42}$. Les notes qu'on trouve si fréquemment servir de sous-titre sur les couvertures des cahiers apportent un second éclairage sur le contenu du journal. Dans le cas d'un journal viatique comme celui de la "Campagne à Vienne", qui nous concerne plus directement, dès la première entrée de son journal, le titre courant differe du titre principal du journal, «Puérils Mémoires de mon voyage en Allemagne». Ce titre met l'accent sur l'écriture personnelle avec la pointe

32. J, p. 517 : "Campagne de Vienne en I809" R5896, t. V, fo $116 v^{\circ}-96 v^{\circ}$ (cahier relié à l'envers).

33. J, p. 539.

34. J, p. 827.

35. J, p. 87 I.

36. J, p. 335. Mais aussi «Journal de sa vie depuis le I5 avril I806 jusqu'au 3 mai I8Io. Il y a pour ce temps trois autres cahiers et quelques feuilles détachées pour la Campagne de Vienne en I809." (p. 427)

37. "Je m’en veux de n'avoir rien écrit depuis mon retour. Ce fut le 27 novembre." ( $J$, p. 82I)

"De tout ce que dessus je conclus que ces trois mois-ci ont été les plus constamment happy of my life.» ( $J$, p. 822)

38. J, p. 578. Mais aussi : "Djorn'l from the I6th August I8Io till the [un blanc].» (p. 624)

39. J, p. 473 .

40. J, p. 9I0.

4I. On peut aussi citer d'autres journaux : "Journal du is février I8IO au [un blanc]» (p. 548), "Journal commencé le 4 février I8I3, à Paris, rue Neuve-du-Luxembourg, $\mathrm{n}^{\circ} 3$ " (p. 835), "Journal du is février i8io au [un blanc]» (p. 548).

42. Brouillage dont témoigne la remise en cause actuelle de la séparation opérée par V. Del Litto dans ses éditions respectives des Euvres intimes et du Journal littéraire. 
d'autodérision nécessaire à toute écriture du Moi-Je. D'autre part, dans une note ultérieure datant de I8I3 et figurant sous le titre lui-même, Stendhal précise : "Ceci n'est qu'un journal destiné à m’observer moi-même, nullement intéressant pour les autres.» Une mise en garde parmi tant d'autres mais qui replace le journal de voyage dans une nouvelle perspective, analytique.

Si tous ces éléments «invitent à penser que Stendhal, à l'époque où il tient son journal, conçoit cette activité en termes de «journal de voyage ${ }^{43}$ », il devient difficile de songer à confronter le journal de séjour au journal de voyage. Toutefois, on trouve aussi chez Stendhal une acception plus littéraire du terme.

\section{Le journal de voyage et le voyage : une écriture sous influence littéraire}

Pour Stendhal, un journal de voyage "accompli ${ }^{44}$ » est un journal nourri de «renseignements». Il écrit à Faure, depuis Sankt-Pölten, le Io mai I8o9 : «Réunis, je t'en prie, tous les renseignements qui peuvent servir à un j[ourn] al de mon voyage. Je ferai copier cela par quelque écrivain du coin des rues, bien bête et ayant une belle plume. Le temps me manque pour tout ${ }^{45}$." Se profile ici un travail de réécriture qui évoque la façon dont il voudrait refaire le Nouveau Voyage d'Italie de Misson : effacer d'un trait de plume la moitié $\mathrm{du}$ livre et compiler les plus grands esprits qui ont écrit sur le sujet ${ }^{46}$. C'est, peut-être en ce sens qu'il faut comprendre sa tentative d'écrire son "Voyage à Brunswick" sous une forme hybride qui est celle du récit de voyage - elle emprunte des atours diaristes mais s'organise selon des chapitres thématiques.

Ce sont précisément des Voyages que Stendhal lit pour préparer sa Campagne en Autriche. Dans l'Europe romantique, désormais «le livre préexiste au voyage, de sorte que se renverse la fonction sémiotique" (c'est le paysage, confronté au livre, qui permet de lire ce dernier)", écrit Chantal Massol ${ }^{47}$. Stendhal ne fait rien d'autre, lui qui lit, avant le départ, jusqu'au bout mais en haussant les épaules le Voyage de John Carr dans le $\mathrm{Nord}^{48}$ et sur les routes,

43. S. Ceysson, "Le "journal intime" de Stendhal», art. cité, p. 5I.

44. J, p. 878 .

45. À Faure, S[ank]t-Pölten, Io mai I809 (p. 830).

46. J, p. 878 .

47. Ch. Massol, "Conclusion", dans Voyager en France au temps du romantisme : poétique, esthétique, idéologie, textes réunis par A. Guyot et Ch. Massol, Grenoble, ELLUG, 2003, p. 39I.

48. Le 3 février I809, Stendhal juge sans concession le voyageur anglais John Carr, auteur d'un ouvrage traduit en français sous le titre L'été du Nord ou Voyage autour de la Baltique par le Danemark, la Suède, la Russie et partie de l'Allemagne, dans l'année I804, et publié en I808: "J'ai lu hier et aujourd'hui le voyage de John Carr dans le Nord; coup d'œil rapide d'un homme d'esprit, mais qui le cherche, et qui a pour principes ceux de la société. Ce livre, fait 
le Voyage de John Moore en France, en Suisse et Allemagne qu'il appelle simplement Voyage in Germany ${ }^{49}$. Ce voyage lui sert de contre-point intéressant par rapport à sa propre expérience. Ainsi se réjouit-il alors qu'il parcourt une route pittoresque le ramenant à Neustadt de prendre "plus de plaisir qu'un jeune Anglais riche voyageant par cette même route. Je partais pour ce raisonnement du voyage de Moore, que je lis avec plaisir ${ }^{50}$ ". Les Voyages, genre en vogue au XIX ${ }^{e}$ siècle ${ }^{\S I}$, offrent ainsi à Stendhal une possibilité d'apprivoiser, dans une certaine mesure, sa Campagne à Vienne. Mais rien ne l'a préparé au spectacle de l'horreur du pont d'Ebersberg; reste la littérature qui lui sert toujours de dérivatif. Ce jour-là, il demande à Faure de lui envoyer Les Martyrs: "J'ai besoin d'imagination ${ }^{52}$ ", écrit-il simplement. En lisant les témoignages des mémorialistes et épistoliers de la grande armée, Nathalie Petiteau reconnaît qu' «il fallait [...] toute l'ironie et la sensibilité d'un Henri Beyle, pour qualifier ces campagnes de voyages ${ }^{53}$ ».

Le journal de voyage apparaît donc comme un genre littéraire pris entre deux tenailles, celle d'une forme diariste ouverte, et celle d'un livre achevé; d'ailleurs Stendhal entrera en littérature par ce biais. Difficile donc pour le journal de séjour de se poser en s'opposant face à une forme à la fois si présente et si diverse dans l'œuvre stendhalienne. Reste donc à faire surgir des limbes cette réalité par une étude précise des journaux eux-mêmes, approche qui a l'avantage du plaisir du texte.

avec de l'esprit français, mais qui n'en a nullement la partie brillante (les Lettres persanes, la Vie du c [om] te de Gramont, etc.) plaît ici. Il nous fait souvent hausser les épaules, mais l'on va au bout. Louangeur à toute outrance.» ( $J$, p. 515)

49. Il évoque les «cent pages de Moore sur la cour de Vienne» ( $J$, p. 52I) qu’il lit à son arrivée à Ulm, bien ennuyé par la pluie et le froid qui l'ont accompagné jusqu'à cette étape. Dans une lettre à Faure datée du 26 avril ı809, il évoque non sans ironie comment il se trouve "tranquillement dans [une] chambre superbe, mais sans feu et sans lit, à feuilleter le Voyage de Moore in Germany" (À Félix Faure, Landshut, 26 avril ı809, CG, p. 826).

50. Landshut, 24 avril I809, J, p. 529.

5I. Jean-Paul Dufief note qu'en ce XIX ${ }^{e}$ siècle où la mode est à la littérature viatique, la lettre de voyage qui peut séduire un large public sert de modèle à un genre littéraire qui connaît une vogue encore plus grande que les journaux de voyages (J.-P. Dufief, "Présentation ", dans La lettre de voyage [actes du colloque de Brest, I8, I9 et 20 novembre 2004], textes réunis et présentés par J.-P. Dufief, Presses universitaires de Rennes, 2007, p. Iо).

52. À Faure, Wels, 3 mai 1809, $C G$, p. 829.

53. N. Petiteau, «Les voyages des hommes de la Grande Armée : de la vie militaire aux pratiques de la mobilité géographique» dans Voyager en Europe de Humboldt à Stendhal. Contraintes nationales et tentations cosmopolites I790-I840, N. Bourguinat et S. Venayre (dir.), Nouveau Monde éditions, 2007, p. 362. 


\section{Journaux et correspondance : le séjour viennois de Stendhal se dédouble}

À Vienne, Stendhal écrit un bref journal et tient une correspondance active avec sa sœur. Rien d'étonnant en soi, si ce n'est l'étanchéité absolue de ses deux journaux de séjour. L'écriture diariste se concentre sur le seul mois de novembre, sur un seul sujet- sa relation avec Madame Palfy, avec une seule fonction - l'analyse. L'écriture épistolaire, quant à elle, couvre les six mois, y compris celui de novembre, mais il n'y est pas question du séjour de Madame Palfy. Certes, Stendhal avait déjà évoqué dans une lettre à Pauline sa passion pour celle qu'il appelle alors Elvire ${ }^{54}$; mais avec l'arrivée de la femme aimée, la correspondance se vide de toute confidence amoureuse. Dès lors, les deux passions éprouvées à ce moment-là par Stendhal - l'amour et l'ambition trouvent deux formes d'expression spécifiques. À travers cette répartition des sujets, ce n'est pas la question de l'«intime» qui se joue - confidences et ouverture du journal en témoignent - mais celle des différentes fonctions attribuées à l'écriture diariste et épistolaire.

\section{Le journal de séjour épistolaire : de la primesauterie à l'ambition}

On sait la porosité des genres des journaux et de la correspondance chez Stendhalss, mais il faut souligner avec Brigitte Diaz combien chaque genre conserve pour Beyle une certaine spécificité : «on trouve relativement peu de récits dans la correspondance de jeunesse, et quand il y en a, c'est en général pour parer aux défaillances du journal qui ne peut pas être tenu pour des raisons contingentes ${ }^{56}$.» C'est le cas lors de l'incendie de Moscou que Stendhal décrit longuement à Pauline :

Remets ce bavardage à Félix qui en fera collection. Il faut au moins que je tire ce parti de ces plates souffrances, de m'en rappeler le comment ${ }^{57}$.

C'est aussi le cas lors de la Campagne à Vienne. Stendhal se sent incapable de rédiger son journal dans la promiscuité du bivouac et utilise le relais de la lettre pour transmettre à Faure les annales de sa vie de soldat : "J'ai eu l'idée d'écrire mon journal le plus possible, et de t'en envoyer les feuilles à mesure;

54. À Pauline, Vienne, I4 juillet I809, $C G$, p. 835 .

55. "J'avais le projet de te faire une grande lettre, ensuite un journal de tout ce que je vois et de mille petites chose que je sens et que tu aurais senties aussi.» (À Pauline, [I9 avril I809], CG, p. 82I)

56. Br. Diaz, Stendhal en sa correspondance ou "L'Histoire d' un esprit», Honoré Champion, 2003, p. 199.

57. À Pauline, 4 octobre I8ı2, CG, t. II, p. 362. Consigné dans le journal à la date des I4 et I5 septembre I8I2. 
deux avantages : abréviation de lettres et sûreté. Seulement, ne perds pas les feuilles ${ }^{5}$.» Selon la critique, journal comme correspondance ont des fonctions assez distinctes. De façon théorique, «le journal se veut autant radioscopie du corps que de l'âme, compte rendu quasi médical d'une existence quotidienne. En cela, il récuse comme inadéquat tout artifice rhétorique, tandis que la lettre reste quelque part, malgré tout, une écriture de la séduction, où le moi s'exprime et s'étudie à travers le prisme d'un style ${ }^{\varsigma 9}$ ". Stendhal le reconnait, lui qui écrit dans son journal au sujet d'une des lettres qu'il a envoyées à Pauline :

J'ai décrit les sensations et événements antérieurs à Burghausen dans une lettre de huit pages à ma sœur. Ça manque de profondeur et est enjolivé. Je l'ai portée jusqu'ici pour faire mon journal d'après elle, mais je n'ai pas eu le temps ${ }^{60}$.

Il s'agit d'une longue lettre racontant toutes les difficultés rencontrées et surmontées pour trouver un logement à Alt-Eting, puis le départ précipité dans la nuit pour Burghausen. Tout un arsenal rhétorique assaisonne la lettre. Stendhal y manie l'hyperbole ${ }^{61}$ et la clausule rythme ternaire ${ }^{62}$, la comparaison et l'art de la dramatisation ${ }^{63}$.

Dans le massif de lettres envoyé à Pauline depuis Vienne, on observe une certaine esthétique de la primesauterie : "Adieu ma lettre est bien décousue», écrit-il à la fin d'une lettre à Pauline où il passe d'un sujet à l'autre sur un mode qui s'apparente à une conversation ${ }^{64}$. Dans ses lettres, il fait allusion à de multiples amours, car, pour reprendre la formule salée de Philippe Berthier, "côté filles, la capitale autrichienne est un séjour délicieux : à chaque pas on tombe sur une jolie femme et Henri dira sans fard que là-bas c'est le cul qui lui a donné le plus de plaisir ${ }^{65}$ ». Il est question de "filles ${ }^{66}$ " au pluriel, d'une passion un peu mystérieuse pour laquelle, un jour, il a des spasimi, et qui s'arrête brutalement ${ }^{67}$; d'une autre, clairement identifiée cette

58. À Félix Faure, I6 avril I809, CG, p. 82I.

59. Br. Diaz, ouvr. cité, p. 200.

6o. Enns, le 5 mai I809, J, p. 532.

6I. "Je me mis donc à menacer tout au monde, même les gros nuages noirs qui me couvraient de versées épouvantables. (À Pauline, Burghausen, 29 avril ı809, CG, p. 827)

62. "Tout le monde menaçait, jurait, criait, dans cette exécrable petite chambre.» (Ibid., p. 825$)$

63. "Nous nous sommes réveillés ce matin vers les cinq heures, nos chevaux allant le galop à une descente. Nous avons crié comme des aigles, fait arrêter et mettre le sabot. " (Ibid., p. 828)

64. À Pauline, Vienne, 25 juillet I809, CG, p. 838-839.

65. Ph. Berthier, Stendhal. Vivre, Écrire, Aimer, Éditions de Fallois, 20Io, p. I72. Il cite un passage du Journal, 7 juin I8Io, p. 593.

66. À Pauline, Vienne, I5 juin I809, CG, p. 834.

67. À Pauline, V[ienne], 4 septembre I809, $C G$, p. 842 . 
fois, pour Babet Rothe ${ }^{68}$ - «actrice-chanteuse, décidément figure fétiche de la libido beyliste», qui le rend jaloux ${ }^{69}$, lui fait craindre l'art de plaire aux femmes de Montbadon ${ }^{70}$, l'entraîne presque à se battre en duel et dont "une petite maladie [1]'empêche[ra] de profiter ${ }^{71}{ }^{\prime}$. $S^{\prime}$ il n'ose écrire en toutes lettres le nom de la maladie de Vénus dans la lettre à la sœur, il dévoilera le pot aux roses sur le cahier de la "Consultation pour Banti» dans une note datée de I819: «il s'agissait de la vérole; rien moins que cela ${ }^{72} . »$

Mais ce ne sont pas les femmes, toujours évoquées allusivement, mais l'ambition qui occupe durablement la correspondance de Stendhal. Adressées à Pauline, trois lettres de Vienne et une de Sankt-Pölten sont consacrées à cet "accès d'ambition qui ne [lui] laisse de repos ni jour ni nuit ${ }^{73}$ " à partir d'octobre quand il aperçoit la possibilité d'être fait auditeur ${ }^{74}$. Sa correspondance n'a plus alors qu'un seul objet et qu'une seule modalité : l'exhortation; elle devient le journal d'un ambitieux. Le séjour à Vienne est perçu comme un tremplin vers un ailleurs, et Pauline, confidente, devient courroie de transmission, avocat et factotum. Chaque lettre ânonne les mêmes recommandations, se gonfle d'autres lettres non écrites, d'une fortune inventée, de l'appui de personnes dont il ignore si elles sont encore vivantes. C'est à une véritable mystification qu'assiste le lecteur: Stendhal transforme ainsi ses 6.530 livres de rente en "7.530, ce qui aura l'air plus naturel" ${ }^{75}$ ", puis en " 7.556 fr», mais il faudra le dire "avec toute l'effronterie possible ${ }^{76} »$, pour finir par annoncer "7.650 fr", comme « the thing the best true ${ }^{77}$ » Outre l'intérêt réel de cet enjolivement de sa fortune, ce qui transparait clairement, c'est le plaisir

68. Il l'évoque sous la lettre «B.» dans une lettre à Pauline (4 septembre ı8०9, $C G$, p. 842). Il y fera allusion dans son journal le 26 avril I8Io, quand on lui annonce qu' elle pourrait venir à Paris : «l'espoir de revoir ses beaux yeux $e$ l'angelico sembiante [le] pén[è] $\operatorname{tr}[\mathrm{e}]$ d'une vive volupté» (J, p. 570).

69. " [...] j'ai aujourd'hui la vie du plus malheureux des tyrans, rongé par la jalousie la plus noire et la plus humiliante, sans avoir eu un instant pour respirer» (À Pauline, Vienne, 6 août I809, $C G$, p. 840).

70. Dans son journal, Faure écrit : "Après déjeuner, visite à Mlle Ba[bet]. Il paraît que généralement M. de Montbadon inspire peu de confiance.» (Mardi, 8 août I809, J, p. I052) C'est le fameux Montbadon, adjoint aux commissaires des guerres, dont Stendhal disait qu'il "possédait si bien [le] talent de plaire [aux femmes] (À Faure, I8 mai I8o9, CG, p. 834).

7I. À Pauline, V[ienne], 4 septembre I809, CG, p. 842. Stendhal "Côté filles, la capitale autrichienne est un séjour délicieux : à chaque pas on tombe sur une jolie femme et Henri dira sans fard que là-bas c'est le cul qui lui a donnée le plus de plaisir. Avec les risques inhérents à ce qu'on n'appelait pas encore le tourisme sexuel : une chaude-pisse et deux véroles.

72. J, p. I069.

73. À Pauline, Vienne, I4 juillet I809, $C G$, p. 835.

74. À Pauline, V[ienne], I4 octobre [I809], $C G$, p. 844.

75. À Pauline, Vienne, I8 octobre I809, $C G$, p. 845.

76. À Pauline, 26 novembre [I809], $C G$, p. 846.

77. À Pauline, Sankt-Pölten, 7 décembre I809, CG, p. 850. 
d'une facétie qui ne trompe personne. Ce journal de séjour épistolaire trouve donc une unité tardive autour d'un objet unique, l'ambition, qui, au fil de chaque lettre, s'invente, se nourrit et fabrique une destinataire héroïque, omnipotente, au service et à la mesure du moi. Ces dernières lettres du séjour à Vienne sont donc entièrement tournées vers les promesses de l'avenir et ne reposent que sur la bienveillance de la cara sorella.

\section{Le journal de séjour diariste : sismographe d'une liaison platonique}

À l'inverse, le journal de séjour diariste ne relate que le séjour de Madame Daru à Vienne et affiche clairement sa vocation : aider Stendhal «à se former dans quelques années, une idée de $[s]$ a conduite ${ }^{78}$ ». Sachant bien que «l'analyse indiscrète du journal nuit au bonheur ${ }^{79}$ ", il n'entame la rédaction que poussé par sa bashfulness, cette timidité qui le paralyse. Le journal peut, semblet-il, jouer un véritable rôle thérapeutique pour son rédacteur. Stendhal suppose que la consignation précise de ses faits et gestes pourra lui permettre de gagner en hardiesse, de rompre les froids des tête-à-tête, de prendre le ton de la galanterie, et qui sait?, d' "être heureux ${ }^{80}$ ". Aussi son journal peut-il se lire comme une longue suite d'interprétations de signes. Ce n'est pas la première fois que Stendhal courtise Madame Daru : il l'a rencontrée à Brunswick et à chaque nouvelle entrevue il retombe sous le charme de la conquête. Dans le journal de son séjour à Paris en I809, il note scrupuleusement ses invitations à dîner et comment elle «le comble de bontés ${ }^{81} »$. Mais le moment de grâce ne dure pas, Stendhal se sent brusquement dédaigné; il écrit à Faure que les trois derniers jours avant son départ : «elle [le] regardait comme on regarde un baril de poudre ${ }^{82} »$.

Le journal de séjour stendhalien à Vienne se fait donc sismographe des variations d'humeur de Mme Palfy, des secousses de l'âme du diariste, de la température de leur rendez-vous (les fameux froids!). Le journal n'est pas "baromètre de l'âme ${ }^{8_{3}}$ ", mais "baromètre d'une relation" chaotique ou supposée telle. Très rares sont les moments de simples constats : "Son premier abord fut exactement tel qu'il aurait été pour un autre ${ }^{84}$.» Quasiment toutes les phrases font l'objet de modalisation, et sont donc soumises à une

78. J, p. 546 .

79. 28 août I8I2, J, p. 827 .

8o. J, p. 547. Au sens sexuel du terme.

8I. J, p. 517.

82. À Félix Faure, Strasbourg, 5 avril I809, CG, p. 819-820.

83. Référence à l'ouvrage de P. Pachet, Les Baromètres de lâme. Naissance du journal intime, Hachette Littératures, coll. «Pluriel», 200 I.

84. Vienne, 2I octobre I809, J, p. 540. 
interprétation : on trouve tour à tour les verbes "sembler ${ }^{85}$ ", "paraître ${ }^{86}$ ", " $\operatorname{prouver}^{87}$ » / montrer $^{88}$ " / « $\operatorname{voir}^{89} »$, « croire $^{90}$ ", « devoir ${ }^{91}$ » les adverbes «sans doute $^{92}$ ", "peut-être ${ }^{93}$ ". À ce titre, la dernière scène est un chef-d'œuvre du genre. Dans l'urgence du départ, tout fait signe et fait sens : des gants, un baisemain, un bras pressé et le baiser d'adieu. Alors que l'attente se prolonge, elle lui tend la main «avec beaucoup de grâce et peut-être même de la tendresse»; lui-même, «du[t] avoir de la grâce et du sentiment ». On annonce les chevaux : «[il] serre [le bras] qu'elle [lui] a donné, elle s'est sans doute aperçue de ce mouvement». Au moment du départ, il l'embrasse et son baiser «fut donné avec âme et reçu sans froideur, à ce qu'il [lui] parut».

Le diariste juge aussi sa conduite de l'instant et repère les «sottise[s]» commises, que ce soit une simple "faute d'usage ${ }^{94}$ » ou un "grand tort»-comme «ne pas avoir pris au commencement ce ton galant qui permet de tout hasarder ${ }^{95}$ ». Un même constat revient toujours : sa «maudite ${ }^{96} »$ bashfulness le laisse bien embarrassé en tête-à-tête : "J'avais de belles choses à dire, et je ne dis rien ${ }^{97 . » ~ " C e l a ~ a p p e l a i t ~ u n ~ c o m p l i m e n t ~ b i e n ~ n a t u r e l ; ~ j ' e n ~ v i s ~ l a ~ p l a c e, ~ e t ~ n e ~ l e ~}$ fis $\mathrm{pas}^{98}$.» Du constat d'échec découle enfin, la leçon à tirer; le blâme devient exhortation pour l'avenir : le ton se fait conquérant. C'est là qu'apparaît le mieux l'utilité directe du journal : «Tu ne dois faire de journal qu'autant que cela peut t'aider à vivre da grande ${ }^{99}$.»

Il ne faut pas manquer, si je la revois, de prendre le ton d'une galanterie gaie dès le premier abord. Un peu plus de hardiesse de plus, et nos nombreux tête-à-tête,

85. Voir l'incise : "ce me semble» (p. 541), le présentatif «Voilà ce me semble» (p. 542); le verbe impersonnel «Il me semble évident que» et le verbe personnel : «elle sembla faire comme exprès» (Novembre [I809] ( $2^{\mathrm{e}}$ entrée) p. 542).

86. «Mon amabilité a paru faire plaisir à Mme Z.» (J, p. 545)

87. "[...] de l'amabilité dont elle est avec tout le monde, qui prouve l'absence de tout autre sentiment».; "avec une voix altérée et presque les larmes aux yeux, qui prouve [...]» (J, p. 54I).

88. "Ce trait montre à la fois ce qui manque à mon caractère, et la disposition que je lui suppose (à elle).» (, p. 544)

89. "The foot, the hand. Je ne vois rien de marqué à mon égard." (J, p. 544)

90. "[...] je la crois contente de ses courses, mais peu de son compagnon» ( $J$, p. 543).

91. "Je dus avoir de la grâce et du sentiment, car j'étais animé, pas trop animé, et je ne me contraignais nullement. » (J, p. 546)

92. «Les spectateurs croient peut-être qu'elle m'aime, les sots vont peut-être jusqu’à croire que je l'ai.» (J, p. 546)

93. "[...] avec beaucoup de grâce et peut-être même de la tendresse» (J, p. 546).

94. 2I octobre I809, J, p. 540 .

95. J, p. 543.

96. J, p. 545 .

97. $J$, p. 544 .

98. J, p. 543

99. da grande $=[$ en grand homme]. (23 septembre $1813, J$, p. 888) 
qui ont dû lui paraître froids, eussent été charmants, car la glace rompue, je suis sûr de moi en cela, j'eusse paru dans tout mon avantage ${ }^{100}$.

Le ton impératif, la certitude, la redondance témoignent de cette méthode Coué qui apparaît en filigrane dans tout le journal : «avec un ton plus résolu, je prenais [cette place] et acquérais une prépondérance immense $\mathrm{e}^{\mathrm{ror}}$." Mais si Stendhal se promet une conquête future, il fait aussi de son journal le lieu de la réalisation de son fantasme. Rien de plus évident à ce titre que la mystification finale avec l'indication «Parenthèse» qui ouvre l'espace de l'affabulation : "J'ai perdu les journaux contenant la suite et la fin. Tout cela se termina en six minutes, deux mois après, et je l'aie eue un an de suite, six fois par semaine. Parenthèse $\mathrm{I}^{\mathrm{IO2}}$.»

\section{Relecture et réécriture : "La Consultation pour Banti»}

En I809 la Campagne de Madame Palfy n'est pas finie - Stendhal n'est pas tombé à Vienne. Aussi n'est-il pas étonnant de le voir revenir sur cet épisode dans sa "Consultation pour Banti", rédigée le 3 avril I8II, lorsqu'un nouveau siège se prépare. Plus étrange est la relecture du séjour qui y est faite. Dans la Consultation, les faits et gestes de Madame Daru, objets de longues ratiocinations dans le journal, deviennent soudainement limpides. C'est que la relecture du séjour est en même temps une réécriture de l'histoire. L'accueil que lui réserve Madame Daru à Vienne n'est plus «exactement tel qu'il aurait été pour un autre ${ }^{\mathrm{I03}}$ " mais "d'un intérêt tendre $\mathrm{d}^{\mathrm{I04}}$ ». La Consultation dramatise leurs relations : ainsi, c'est "avec inquiétude ${ }^{\text {ros }}$ " que Mme Daru interroge Stendhal sur sa santé. Elle "s'empare ${ }^{\mathrm{TO} 6}$ " littéralement de lui pour visiter la ville "les monuments, les promenades et les environs de Vienne», ce que le journal - très elliptique - n'évoque guère. Cette réécriture du séjour viennois n'est guère modalisée : il n'y a plus place pour l'interprétation, le cours des ans est sans appel. Une seule fois apparaît le verbe «sembler" : "Il me semble que, pendant tout ce séjour à Vienne, la duchesse fit à Banti autant d'avances qu'en comporte le caractère que nous lui supposons ${ }^{107}$.» $\grave{A}$ travers cet exemple apparait bien le dispositif énonciatif complexe de ce texte qui est écrit par Crozet sur la demande de Stendhal mais aussi sous sa dictée, comme

Ioo. J, p. 546.

IOI. J, p. 54I.

IO2. J, p. 547.

I03. J, p. 540.

I04. Ibid.

I05. Ibid.

Io6. Ibid.

I07. J, p. 1069. 
le pronom «nous» le laisse bien entendre - des traces de correction, ajouts et biffures de la main de Stendhal témoignent aussi de la rédaction collaborative de ce texte. Stendhal juge et partie de sa propre Consultation n'hésite donc pas à réécrire la fin de l'histoire : «elle lui donna mille occasions de se déclarer, était toujours seule avec lui, avait l'air tendre, etc. et, après trois mois de séjour, l'embrassa à son départ avec expression et en le serrant dans ses bras $^{\mathrm{ro8}}$ ». Ce serait donc Alexandrine qui aurait fait le premier pas. À lire ce texte, si Stendhal n'a pas été "heureux" à Vienne, c'est moins à cause de sa fâcheuse timidité que d'un profond aveuglement. Dans une note qu'il ajoute en I8I9, Stendhal apporte encore un nouveau regard sur ce séjour. "Avis. Banti fut timide, amoureux de l'amour sans le savoir, il s'arrêtait à chaque pas pour jouir et ainsi la manqua par bêtise, et faute d'attaquer. I8I9 ${ }^{109}$ ". À cette époque, Stendhal rédige De l'Amour et cette réflexion en est tout imprégnée. Aimer l'amour, c'est retarder sa réalisation, c'est préférer ce qui la précède, tous ces atours qui font pressentir qu'on est aimé. Dans le chapitre «De l'intimité» de De l'Amour, Stendhal écrit que «le plus grand bonheur que puisse donner l'amour, c'est le premier serrement de main d'une femme qu'on aime ${ }^{\mathrm{IIO}}$ ». À chaque relecture, c'est sous un nouveau jour que la conduite de Stendhal-Banti apparaît tandis que le journal de séjour stendhalien à Vienne s'ouvre et se ferme - métaphoriquement tout au moins.

Ainsi, le double journal de séjour stendhalien met-il déjà en évidence la complexité de cette forme de journal. Écriture "prisonnière» d'un lieu, d'un temps, ou d'un sujet, le journal de séjour est souvent dialogue, ouverture vers autrui, aspiration vers l'avenir ou l'ailleurs. L'arrivée de Félix Faure apporte à Stendhal une diversion agréable dans sa vie d'expatrié. Le journal de l'ami offre un regard nouveau sur le séjour stendhalien, mais aussi un œil neuf sur une ville dont on peut mieux cerner les contours mouvants. Quelle représentation de Vienne offrent donc les «journaux croisés ${ }^{\mathrm{III}}$ » de Stendhal et de Faure? Plus que l'image de la ville, c'est le regard qu'ils portent sur elle qui nous intéressera, Vienne devenant un miroir du moi.

108. J, p. 1070.

I09. Ibid.

IIO. De l'Amour, ouvr. cité, p. Io9.

III. Lexpression est reprise à Ph. Lejeune qui consacre un chapitre aux «journaux croisés" dans son ouvrage Le journal intime. Histoire et anthologie écrit en collaboration avec C. Bogaert : Deux cas sont distingués : « [Les journaux croisés] peuvent l'être, après coup, par la lecture que nous faisons de journaux parallèles tenus par des personnes qui ont partagé une même expérience, ou vécu ensemble une partie significative de leur vie. Ils peuvent l'être, dès l'origine, par deux personnes qui conçoivent un projet de journal commun, tenu "à quatre mains"” (Textuel, 2006, p. 134). 


\section{Vienne sous le regard des journaux croisés de Stendhal et Faure}

Vienne signe les retrouvailles de Stendhal et de Faure. Mais les deux amis ont-ils vraiment séjourné dans la même ville? Quelques lieux remarquables se retrouvent d'un journal à l'autre et forment les îlots de la topographie viennoise : le Prater dont la renommée est unanime "délicieuse promenade, dit-on ${ }^{\mathrm{II}}$ " "la plus belle promenade de l'Europe, disent ceux qui peuvent en juger ${ }^{\mathrm{II} 3}$ ", le bastion ${ }^{\mathrm{II} 4}$, le théâtre de la porte de Carinthie ${ }^{\mathrm{II}}$ et celui de la Vienne ${ }^{\mathrm{II}}$, des jardins ${ }^{117}$, l'église Saint-Étienne (sur laquelle ils n'ont pas le même jugement). Pour Stendhal, c'est une «magnifique église gothique, non pas réparée à neuf comme la cathédrale de Reims, mais laissée avec son vénérable gris noir, comme celle de Strasbourg ${ }^{118}$ ». Alors que Faure note sèchement : "Vu l'intérieur de la cathédrale (Saint-Étienne) qui n’a rien de remarquable; style gothique; beaucoup de tombeaux, d'inscriptions ${ }^{\text {II)." }}$

Néanmoins, il faut noter que les deux amis n'envisagent pas la ville sous le même angle, ce qui s'explique bien sûr par le caractère différent de leur séjour : séjour libre de toutes contraintes de l'un - Faure craint seulement la colère du père, et séjour forcé de l'autre - Stendhal est soumis aux aléas des campagnes napoléoniennes. Faure, qui n'est à Vienne que pour un mois, visite activement la ville et tous ses monuments remarquables. Il se comporte en touriste et en amateur d'art :

je suis allé visiter tous les jours [l'admirable mausolée de Marie-Christine (œuvre de Canova)], et un moment que je serai disposé j'essaierai de le décrire pour me le rappeler, ou de m'en procurer une gravure ${ }^{120}$.

Selon les analyses de Gérald Rannaud, c'est l'époque où l'art devient une pratique culturelle discriminante :

Le voyage, comme occasion d'une pratique de l'art et d'une appropriation symbolique des grands monuments et des hauts lieux du patrimoine, se donne désormais comme moment et signe de consécration, de confirmation, de promotion ${ }^{121}$.

II2. Lundi 7 août, I809, J, p. IOSI.

II3. À Pauline, [Vienne, septembre I809], $C G$, p. 843.

II4. Dimanche, 6 août I809, $J$, p. Ioso.

II5. À Félix, I8 mai I809, $C G$, p. 833.

II6. Dimanche, 6 août I809, J, p. I050.

II7. Voir «le petit jardin», (Dimanche 6 août I809, J, p. I050) ou «les jardins Razoumovsky»

(À Pauline, [Vienne, septembre I8o9], CG, p. 843).

II8. À Pauline, Vienne, 29 novembre I809, $C G$, p. 847.

II9. Dimanche, 6 août I809, J, p. I050.

I2O. Ibid.

I2I. G. Rannaud, art. cité, p. I42. 
Le voyageur se veut aussi esthète et dilettante. Toutefois, le journal de Faure ne se complaît pas dans les descriptions, il prend parfois un tour de compterendu sténographié : "Vu l'intérieur de la cathédrale ${ }^{\mathrm{r22}}$ »/ "Allés ensemble au bastion $^{\mathrm{I23}}$ » / "Visité l'hôpital ${ }^{124}$ ». Stendhal, quant à lui, évoque peu la ville qui sert de simple cadre à ses amours. Les qualifications sont suffisamment vagues et hyperboliques pour ne rien désigner en particulier : "ville très agréable ${ }^{\mathrm{r25}}$ ", "ville charmante ${ }^{\mathrm{I2} 6}$ ", "un des plus beaux climats qu' $[i 1]$ ai $[\mathrm{t}] \mathrm{vu}^{\mathrm{I27}}$ ». De là, le rapport symbolique que Stendhal semble entretenir avec la ville. Tout Vienne se réduit, par un procédé métonymique, au Kahlenberg (le mont Chauve) et le séjour de Madame Palfy se résume à la promenade en ces lieux, moment de climax dans son journal - tant physique que dramaturgique. Stendhal lui-même laisse entendre que «ce jour [de la promenade] a été le zénith de mon crédit auprès de Mme de T[riangi] ${ }^{128}$ ». Son séjour tient en trois jours et l'importance capitale de l'excursion au Kahlenberg est aussi suggérée par le titre figurant sur la couverture de son cahier : une équivalence y est posée entre les lieux et les personnes - «Journal du Kahlenberg et du Leopoldsberg ou (Liaison du colonel L. avec la princesse P [alfy $]^{129}$."

Le rapport à la réalité physique de Vienne diffère donc du tout au tout de Faure à Stendhal. La parade de Schönbrunn ${ }^{\mathrm{I} 30}$, épisode relaté dans le journal de Faure, est un exemple précieux car elle nous offre l'un des rares moments où les regards de Stendhal et de Faure se croisent et se confrontent sur la ville. En effet, pour Faure, la parade forme à la fois un "spectacle curieux ${ }^{131}$ » et une "chose remarquable et belle ${ }^{132}$ ». Il en fait donc une description précise et minutieuse, relevant à la façon des "dénombrements faits par les poètes des armées prêtes à combattre les unes contre les autres" les différentes troupes réunies. Au contraire, Stendhal «ne voyait, lui, que des moutons rassemblés pour être menés à la boucherie ${ }^{133}$ ». Faure ajoute : "Cette idée s'accorde avec sa position et sa tournure d'esprit. Les circonstances me l'expliquent très bien ${ }^{134}$.»

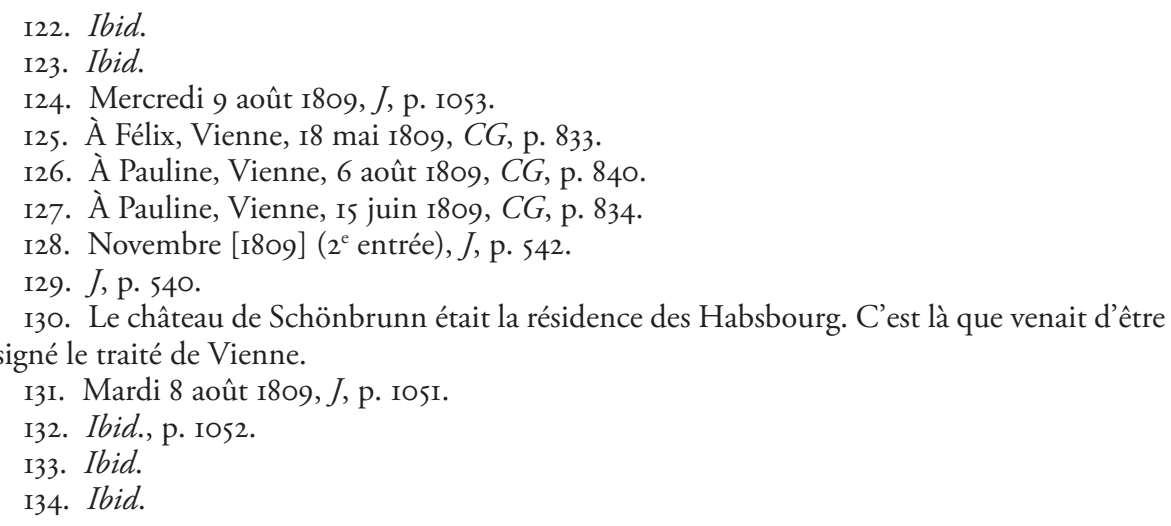


De fait, Stendhal est imperméable à tout romantisme chevaleresque depuis qu'il fait partie de l'armée napoléonienne et qu'il a été confronté aux horreurs de la guerre. Reste qu'à travers cette divergence, apparaissent plus profondément, deux façons de se voir et donc de s'écrire dans la capitale autrichienne.

\section{Vienne ou le fantasme italien de Stendhal}

À Vienne, Stendhal respire dans un premier temps comme une bouffée d'air italien. Il en retrouve le climat, la musique, les femmes. À Landshut, Stendhal écrit : "Cette ville fit sur moi l'impression de l'Italie. J'y vis en demi-heure cinq à six figures de femme d'un ovale beaucoup plus parfait qu'il n'appartient à l'Allemagne ${ }^{135}$." Si c'est en février I809 qu'on trouve la première mention de Mozart ${ }^{136}$, c'est sous le chant de «la belle romance du Figaro ${ }^{137}$ » : Voi che sapete que Stendhal entame la Campagne d'Autriche. Il parle de «musique divine ${ }^{13^{38}}$ " à Pauline et s'il n'aime pas Haydn, il commence à comprendre Don $J u a n^{139}$, et trouve toujours à se réfugier auprès du Matrimonio segreto ${ }^{140}$.

Mais en réalité, Vienne n'offre à Stendhal qu'un succédané d'Italie : certes, la ville offre bien des charmes, mais il n'y retrouve pas l'oisiveté et la faculté de jouir du pays des orangers - dont il offre pourtant un spécimen à sa maitresse! Il doit y travailler "jour et nuit", première restriction, et surtout il souffre d'accès de fièvre ${ }^{\mathrm{I} 4 \mathrm{I}}$, ce qu'il ne cesse de répéter à sa sœur dans toutes ses lettres. Ainsi, à toute caractérisation élogieuse de la ville correspond une restriction, alternance de connotation euphorique et dysphorique : «Le séjour de Vienne me charme et produit une singulière tristesse $[\ldots]^{142}$.» C'est le regret de ne pas pouvoir jouir des jolies femmes à chaque pas que Stendhal exprime ici. Plus

135. $J$, p. 530 .

I36. " [...] j'ai mieux aimé aller à la deuxième représentation de Cosi fan tutte de Mozart. Musique suave, mais c'est une comédie, et Mozart ne me plait que lorsqu'il a à exprimer une mélancolie douce et rêveuse» (4 et 5 février [1809], J, p. 515-516).

I37. [I2-I8 avril I809], J, p. 518.

138. Â Pauline, Vienne, I5 juin I809, $C G$, p. 834.

I39. À Pauline, Vienne, 25 juillet I809, $C G$, p. 839.

I40. À Pauline, Vienne, 6 août I809, $C G$, p. 840 .

I4I. "Depuis mon départ de Paris, j'ai vu beaucoup de choses nouvelles; j'ai eu beaucoup de peines, mais physiques. J'ai enfin accroché quelques accès de fièvre qui m’ont empêché d'aller à la bataille du 6 de ce mois, spectacle à jamais regrettable : cinq cent mille hommes se sont battus cinquante heures.» (Wagram) (À Pauline, Vienne, I4 juillet I8o9, CG, p. 836); "Je souffre toujours de cette fièvre dont je t’ai parlé, mais cela n'influe pas beaucoup sur la situation de mon âme». (À Pauline, Vienne, 25 juillet I809, CG, p. 838) Voir dans le journal de Faure : "I found Henri in his bed and sick. J'assistai à une consultation sur sa fièvre entre son docteur allemand Carino et un chirurgien major de la Garde. À déjeuner, je fus présenté à sa société (M. de Montbadon excepté qui était à la campagne).» (Mercredi 2 août I809, CG, p. I049)

I42. À Félix, Vienne, I8 mai I809, $C G$, p. 834. 
souvent c'est la fuite de l'instant présent qu'il ressent âprement. «Vienne, qui est une ville charmante, glisse sur moi; je n'y vois que ce que j'aime et que je ne puis pas voir $[\ldots]^{143}$." Climat exceptionnel, très bien, mais «il ne manque que le temps d'en jouir ${ }^{\mathrm{r} 44}{ }^{\prime}$, écrit-il. De même, il s'exclame dans une lettre à Pauline : "Cheval, filles et musique divine le reste du temps [où il ne travaille pas]. Malheureusement ce reste est bien $\operatorname{court}^{\mathrm{I} 45}$.» Face à ce sentiment d'une fuite en avant - le temps file et les plaisirs de Vienne s'enfuient, d'engourdissement de la vie, une solution se profile : le voyage!

Ainsi, le séjour viennois est l'occasion chez Stendhal d'une nouvelle cristallisation : le désir d'un nouveau voyage en Italie. À travers les incessants conseils qu'il adresse à Pauline pour l'encourager à partir avant de tomber enceinte, c'est son fantasme qui trouve sa propre expression. Dans quasiment toutes les lettres à Pauline, il ne manque pas de, conjointement, la féliciter de sa «non-grossesse» et de la pousser sur tous les tons à voyager. Parfois interrogatif : "Ne songes-tu point à voir l'Italie? Profite de l'heureux temps où tu n'as pas d'enfant ${ }^{\mathrm{T} 46}[\ldots]$."; "Comment ne viens-tu pas à Paris? Il faut courir avant que la corvée des enfants te saisisse ${ }^{\mathrm{I} 47}$ » ou encore «ne trouves-tu pas que Turin, Berne, Marseille sont bien près de Grenoble? À ta place, il me semble que je chercherais à les voir ${ }^{\mathrm{r} 48}$." Le ton se fait parfois plus impérieux : "Cours, galope, vois Milan, Gênes ou Berne ${ }^{\mathrm{I} 49}$.» Et même péremptoire : "Je voudrais te voir voyager. Vous êtes à la porte de la Suisse et de l'Italie. Profite de ta liberté actuelle ${ }^{150}$." Il faut sans doute voir derrière cette insistance le signe du propre sentiment d'enfermement ressenti par Stendhal qui, à l'inverse de Pauline, ne pouvait pas partir sur les routes et profiter de sa liberté.

Le journal de séjour est ainsi marqué par un fort désir d'évasion hors de la ville et une tension vers l'avenir. Le journal devient projets de voyage, passant $\mathrm{du}$ «tu» au «je». Stendhal écrit le 6 août : «Si nous avons la paix, je verrai l'Italie, ne fût-ce qu'un coin. - J'irai voir Bialowiska; j'ai besoin d'une femme aimée pour chasser le sombre horrible qui m'accompagne partout ${ }^{15 \mathrm{I}}$. À nouveau le 7 septembre, le même vœu l'habite :

Si on fait la paix, j'irai à Naples, à Rome, etc. ne dussé-je y passer que huit jours. J'ai économisé soixante louis pour cela. N'en dis rien encore. La chose faite, on

I43. À Pauline, Vienne, 6 août I809, $C G$, p. 840 .

I44. À Pauline, Vienne, 15 juin I809, $C G$, p. 834.

I45. Ibid., p. 847 .

I46. À Pauline, Vienne, I4 juillet I809, $C G$, p. 837.

147. À Pauline, 26 novembre [1809], $C G$, p. 846.

I48. À Pauline, [Vienne, septembre I809], $C G$, p. 843.

149. À Pauline, Vienne, Is juin I809, $C G$, p. 834.

I50. À Pauline, Vienne, 25 juillet I809, $C G$, p. 838

I5I. À Pauline, Vienne, 6 août I809, $C G$, p. 840 . 
la pardonnera; le projet semblerait un monstre. Dès que je pourrai monter à cheval, je vais être toute la journée par monts et par vaux, pensant à toi dix fois le jour, et désirant te voir agissante. Le repos avec notre caractère est l'avant-garde de la mort ${ }^{\mathrm{t} 2}$.

Cette dernière phrase n'est pas sans évoquer ce qu'il écrit dans sa lettre précédente à sa sœur : "Il faut secouer la vie, autrement elle nous ronge ${ }^{153}$." Aphorisme qui marque à la fois l'affirmation d'une âme voyageuse et l'angoisse de l'immobilisme; d'autre part, la parenté d'âme que Stendhal affirme ressentir avec celle de sa sœur et qu'il souhaite lui voir ressentir également peut expliquer son projet de voyage à deux : "ne pourrais-tu venir en Italie dans le temps que je parcourrai ce beau pays? [...] Quel plaisir de voir l'Italie avec toi ${ }^{154}$ !» Le journal de son séjour à Vienne se transforme ainsi en une «invitation au voyage».

En tout état de cause, le séjour viennois aussi agréable soit-il, appelle nécessairement le voyage pour Stendhal : «Partir de Vienne me déchirera le cœur; mais, quinze jours après, je n'y penserai plus qu'agréablement, surtout en voyageant ${ }^{\mathrm{IS}}$." Toutefois Stendhal ne fera pas avant I8II le voyage qu'il appelle de ses vœux.

\section{Vienne ou un terrain d'observations pour un jeune touriste}

Dans le journal de séjour de Faure, Vienne ne devient pas une invitation au voyage, elle est bel et bien un terrain d'observations. C'est l'objectif que le jeune homme se donne d'emblée pour l'écriture de son journal :

J'étais curieux de voir fonctionner ces immenses machines militaires dont Napoléon était l'âme, d'observer sur les populations vaincues les impressions, les traces du passage de nos armées ${ }^{156}$.

Objectif sur lequel il revient le jour de son départ :

Aujourd'hui départ de Vienne où j'ai passé trente-cinq jours, non pas toujours avec plaisir, mais non sans provision d'observations que je ne puis consigner ici, mais qui mûrissent l'expérience ${ }^{157}$.

Cette fonction qu'il assigne à l'écriture diariste entraîne des réflexions d'ordre anthropologique ou ethnologique. Faure juge l'aspect de la ville, ses habitants et ses habitantes - il y manque «des femmes comme la comtesse de

I52. À Pauline, V[ienne], 4 septembre I809, $C G$, p. 842

I53. À Pauline, V[ienne], 4 septembre I809, $C G$, p. 84I.

I54. À Pauline, Vienne, 25 juillet I809, $C G$, p. 839.

I55. Ibid., p. 838 .

I56. J, p. I049.

I57. Mardi 5 septembre I809, J, p. I057. 
Palf $[y]^{158}$ ", observe-t-il. Il appuie généralement ses réflexions sur une analyse comparatiste, le plus souvent avec la France ${ }^{159}$. Il s'interroge sur les différences de mœurs entre Allemands et Espagnols ${ }^{160}$, et partant du libertinage supposé des femmes allemandes, se demande si la complaisance des hôtesses à son égard traduit les véritables mours allemandes: "Ne serait-ce pas les manières que les Allemands avaient ou ont pour les Français..., maitres du pays?» Mais il évoque aussi longuement une représentation du Don Juan de Mozart.

Le journal de Faure semble correspondre à ce que Stendhal attend d'un journal de voyage, si l'on se reporte à ce qu'il écrit dans son «Voyage à Brunswick». En effet sous l'intitulé "État politique et mœurs» de son chapitre III, il écrit : "La dernière de ces choses est la seule dont l'étude me plaise dans les voyages. Tout ce que je vois ne m'intéresse et je n'en parle que relativement aux mœurs ${ }^{161}$.» Le jugement qu'il porte sur le journal de son ami peut donc apparaître plutôt sévère :

J'ai lu le journal du voyage à Vienne de Faure, j'en ai été content; ses défauts sont style sans couleur, les défauts qu'il montre dans l'écrivain sont propension à ne pas agir, mais aussi candeur et sensibilitét ${ }^{\prime 62}$.

Au-delà de ce que peut avoir d'amusant le reproche de passivité sous la plume de Stendhal, on se souvient de son manque d'audace amoureuse, c'est la critique stylistique qui nous interpelle. Stendhal dit du journal de Faure qu'il est écrit dans un «style sans couleur»; il jugera aussi l'un des siens "trop mal écrit ${ }^{163}$ ». Certes, nous avons déjà relevé combien Faure avait tendance à abréger ses phrases donnant ainsi à son journal de séjour l'aspect d'un compte rendu, on peut aussi supposer qu'aux yeux de Stendhal, son journal manquait d'anecdotes vives et détaillées, comme celle de l'escapade à Vienne racontée à Pauline. Il termine sa lettre par une demande en retour : "écris-moi donc une journée de ta vie. Cela me

I58. Mardi 8 août I809, J, p. I053.

159. "Vienne m'a paru avoir de plus belles maisons que Paris, moins d'édifices remarquables." (Dimanche, 6 août I809, p. I050); "Vienne, jusqu’à ce jour, n’a fait sur moi que l'impression d'une grande ville de province en France [...].» (Lundi, 7 août I809, J, p. I052); "Ce soir, j'ai vu Don Juan au théâtre de Vienne. Ce théâtre est décidément beau [...]. Nous autres Français, nous vantons beaucoup notre Opéra.» (Samedi I2 août, p. I054)

I6o. "Quelle différence entre le caractère des Allemands et celui des Espagnols et des Italiens! Le fond du caractère allemand, c'est bonté, facilité, fidélité. Ce sont d'excellents serviteurs dans tous les genres. Seulement, et ici, les hommes semblent avoir les vertus qui sont d'ordinaire le lot des femmes, tandis que celles-ci ont le libertinage qu'on reproche ordinairement aux hommes.» (Mardi 8 août I809, J, p. I053)

I6I. "Voyage à Brunswick» [1808], J, p. I047.

I62. Lundi I8 juin [18Io], J, p. 599.

163. "Je relis ce journal et le trouve très bon, seulement trop mal écrit, peint, le 2I décembre I8I3, après avoir lu Timon d'Athènes de Shakespeare." (J, p. 867) Il s'agit du "Journal. Commencé le 4 février I8I3, à Paris, rue Neuve-du-Luxembourg". 
charmerait ${ }^{164} »$. Mais on ne dispose que d'extraits du journal de Faure, ce qui ne nous permet pas de porter un jugement global sur son style; dans les deux parties dont nous ne disposons pas, le journal de séjour doit faire place à un journal de voyage qu'il serait intéressant de comparer à la «Campagne de Vienne».

Mais si le style de Faure est jugé fade, on peut noter toutefois la présence d'un langage commun aux deux amis, à la fois dans la présence du pseudonyme "Mme Palfy ${ }^{165}$ " que dans la farcissure des journaux de mots anglais. Certes chez Stendhal, l'anglais semble avoir une véritable fonction cryptographique : il évoque dans cette langue-autre des sujets tabous : l'amour ${ }^{166}$, la politique ${ }^{167}$, il critique ses camarades à mots couverts ${ }^{168}$ ou évoque son moi $^{169}$. Il met notamment à distance sa timidité par un terme anglais plus adapté : sa bashfulness ${ }^{170}$. Chez Faure, l'emploi de l'anglais ne semble pas obéir à des fonctions cryptiques - on le trouve dans des phrases très banales ${ }^{171}-$ mais plutôt à une fonction crypto-ludique, de complicité avec l'autre : il va faire lire son journal à Stendhal. Associant par trois fois l'anglais avec le nom ou la présence d'Henri ${ }^{172}$, Faure marque ainsi une volonté de partage de ce langage métissé, de cet idiolecte stendhalien qui est en fait un sociolecte. Il use d'ailleurs à la fois de l'anglais et de l'italien, réservant, naturellement, ce dernier au domaine

I64. À Pauline, Vienne, 29 novembre I809, p. 850.

165. Faure l'emploie le 8 août I809 (p. I052).

I66. «Le soir, je me mis à écrire vers les in heures, au milieu de quatre camarades ronflant, et je finis, vers les 2 heures, a letter of two pages to milady." (II mai [I809], J, p. 537)

167. "Si j'en puis croire ce qu[e Mme Daru] me dit, M. Z. shall not come to Spain and had for the second time refused the preferment of the g[énér]la D[ejean]. He will live as simple c[sonseiller] of St [ate] and got the next spring to Italy and Swizerland.» ("M. Z. [Daru] n'ira pas en Espagne et a refuse pour la seconde fois la promotion du général D[ejean]. Il veut rester simple conseiller d'État et aller au printemps prochain en Italie et en Suisse.») (Novembre [1809], J, p. 544)

168. "Ầ travers tous ces raisonnements, la route charmante s'écoulait, ce qui y nuisait encore, was the dulness of my partner." (Landshut, le 24 avril I809, J, p. 529)

I69. "Par prudence rien de politique, tous les noms changés; je ne notais que les ob[servati] ons upon myself." ("Campagne de I809 de Strasbourg à Vienne», Vienne, le I4 mai I809, J, p. 539); "The life and sentiments of silencious Harry.» (Novembre I809, J, p. 54I)

I70. "Bashfulness and froid blessé à la Rousseau. I809. Vienne, coming on of lady Palfy." (Vienne, 2I octobre I809, J, p. 540) «Ensuite au clocher de Saint-Étienne. Le tête-à-tête dans ce lieu si sûr m'a fait maudire my bashfulness et, par conséquent a augmenté le froid.» (20 novembre [1809] à 2 heures moins un quart du matin, $J$, p. 545)

I7I. "Rentré at home sans me perdre." (Samedi I2 août, J, p. I055)

172. "I found Henri in his bed and sick." (Mercredi, 2 août I809, J, p. I049); To day I went with Henri to Schönbrunn. (Mardi 8 août I809, J, p. IO5I); «Henri m’avait procuré une feuille de route; nous nous séparâmes au café de Graben Square où nous achetâmes quelques flacons d'essence de rose d'un joli jeune Grec d'une physionomie charmante et belli occhi.» (Mardi 5 septembre I809, J, p. I057) 
de la séduction. Mis en évidence en début ou en fin de phrase, de paragraphe et d'entrée du journal, le langage-autre semble une langue de reconnaissance. Pour reprendre les termes de Jacques Félix-Faure, «[i]ls agissaient comme des néophytes qui, grâce à ce moyen pouvaient se protéger du vulgaire et créer un climat de mystère et de connivence propre aux initiés ${ }^{173} »$. L'étude de ces «journaux croisés» met donc au jour une complicité - dont témoignent aussi la circulation et l'ouverture du journal au regard de l'ami - au-delà de leurs représentations si divergentes du séjour viennois.

Au terme de notre réflexion, une question reste entière. Face à l'écriture du mouvement qu'est le journal de voyage, le journal de séjour est-il synonyme d'immobilisme? À Vienne, Faure arpente la ville en tous sens, trépigne les premiers jours de ne pas pouvoir visiter autant qu'il le désire et regrette ses quinze jours d'immobilisation. C'est que dans un séjour, chaque jour compte : Stendhal sait l'importance décisive qu'a le jour du départ de Madame Palfy - il n'hésite pas à jouer son va-tout. Mais il faut différencier ancrage physique et aspirations personnelles. De même qu'il existe des spectacles à lire dans un fauteuil ${ }^{174}$, on peut faire des voyages immobiles. Les deux amis sont à Vienne mais pensent au futur : Faure craint son père et repart, Stendhal aspire à la paix et rêve de partir. La ville réelle, terrain d'observations pour le jeune touriste, est pour Stendhal ouverture vers le fantasme d'un ailleurs. De plus, les journaux de séjour ne sont pas clos à jamais : ils vont circuler. Une chose au moins rapproche les deux amis, le besoin de partager leurs expériences ce qui rend impropre l'étiquette d'intime qu'on pourrait vouloir leur apposer. Faure fait relire son journal par l'œil d'un expert, Stendhal ouvrira ses propres annales à Crozet dans l'espoir de pouvoir conquérir Madame Palfy. Encore en vain, car le seul conseil à donner était : "Attaque, attaque, attaque ${ }^{175}$ !», écrira-t-il en I8I9. En séjour ou en voyage, «l'état habituel de Stendhal est [bien] la mobilitét ${ }^{176} »$.

173. J. Félix-Faure, Un compagnon de Stendhal, Félix Faure, pair de France, ouvr. cité, p. 56.

174. Référence à la formule de Musset Un spectacle dans un fauteuil (I832).

175. J, p. 1072.

176. V. Del Litto, «Journal de voyage et journal intime chez Stendhal», art. cité, p. Io. 